(C) 1980. The Genetical Society of Great Britain

\title{
GENETIC ETIOLOGY AND CLUSTERS IN A PEDIGREE
}

\author{
E. A. THOMPSON \\ King's College, Cambridge, CB2 1ST
}

Received 14.iv.80

\begin{abstract}
SUMMARY
As a preliminary tool in the investigation of a rare medical trait in a single extended pedigree or small community, some tests of non-random distribution of cases are constructed. The metrics quantifying this distribution are functions of relationship or descent. Rejection of the null hypothesis of random distribution is thus an indication of possible genetic effects, al though this is not the only viable explanation. The methods are applied to a study of lymphoreticu" malignancies in a Newfoundland community. This community has attracted interest by the preponderance of cases, and offers a unique opportunity for a study of the etiology of the conditions. On such a large extended pedigree it may eventually be possible to assess the relative importance of environment, infection and genetics.
\end{abstract}

\section{INTRODUCTION}

OFTEN pedigrees or communities come to the attention of human geneticists because they show a "cluster" of individuals "affected" with some medical condition ("cases"). Genetic analysis of the pedigree data, or data for the families in the community, is then expected to reveal whether the condition has a genetic component, and, if so, what is the underlying genetic mechanism. However, such a cluster may clearly be an artefact of the ascertainment procedure. A large number of affected individuals may simply be the reason a community comes to attention. A close cluster within a pedigree may bear very different interpretations when more data are collected. If no further cases are found, an infection may be a reasonable explanation: if many are found an environmental effect relevant to the whole reference population may be indicated. Even where the pattern of affected individuals throughout an extensive pedigree suggests a particular genetic mechanism, the mechanism to be inferred may be obscured or even biased by the availability of relatives for examination.

These are, of course, widely recognised problems, but there are many practical difficulties in their resolution. There seems to be a need for some objective criterion by which we may answer the question "Is there a cluster in this pedigree?", where by "cluster" we mean not simply an unusual number of cases, but a pattern of distribution which should stimulate further investigation. The basic aim of this paper is to construct such objective criteria, which are not biased by either the total number of cases observed, nor by the particular structure of the pedigree of the population studied. The problem will be discussed with particular reference to the cases of a variety of lymphoreticular malignancies in three adjacent communities in a small area of Newfoundland (Buehler et al. (1975), Marshall et al. (1980), Salmon et al. (1980)). 


\section{INDICES OF ETIOLOGY}

There have been several previous approaches to this, and similar, problems. Most proposed solutions consider the choice of some "control" individuals, who are then compared with the "cases", in some way. For example, in the context of a large population data base, the distribution of the coefficient of kinship between cases, may be compared with that between randomly chosen matched controls (Williams et al., 1979). There are, however, many problems, both in the choice of controls, and in the comparison of the distributions of an interciependent sample of kinship coefficients (Thompson and Roberts, 1980). For what should controls be matched? Should they be chosen randomly, so that they may be related to cases resulting in non-independence between as well as within the observed distributions of kinships? Should they be chosen unrelated to cases, resolving this last problem, but biassing results through conditioning on a perhaps atypical subpopulation? And how can the distribution of kinship coefficients for "randomly chosen" controls be known, other than empirically, via repeated choices-again intrinsically nonindependent since all result from the same paths of descent in the same underlying genealogy of the population? Thus although a fair preliminary indication, no objective statistical test can be simply constructed.

The above problems are magnified in the case of a small community or single pedigree. Here, in order to match for environmental effects, controls must be chosen within the study population, and hence within the population containing the pedigrees. Although kinship coefficients between cases may be compared with those between controls (as is done by Salmon et $a l ., 1980$ ), and also an empirical distribution of pairwise kinship coefficients found, the construction of statistical tests is difficult. The kinship pairs are interdependent - the lines of descent contributing to the kinship coefficients between cases also contribute to those between controls. The passage of genes to the individuals in question may be highly dependent (Thompson, 1979). There are also problems in the choice of controls. Where carefully matched there may be a subjective element or structural restrictions, resulting in them being more uniformly spread within the study population than expected of a random set of individuals. They may be less closely related to cases, and perhaps atypical. Where chosen at random, statistical problems arise; the choice of a particular control in the sibship of a case (for example) may predominate over other effects. The variances of statistics, derived on the basis of such controls, will be large.

In a medical genetics study, the preliminary question is whether the cases are randomly distributed. Beyond this the pattern of distribution may give further insight into underlying genetic mechanisms. Some indices of trait distribution have been suggested by Fain (1978), Smith et al. (1978), Carmelli et al. (1979), while Williamson et al. (1979) suggest a test statistic enabling some hypotheses of genetic etiology to be tested. We shall not go into these approaches since our concern lies first with the hypothesis of a non-random distribution of cases, which will bear further investigation. That the hypothesis is violated need not bear a genetic interpretation, although some of the proposed statistics may point in this direction.

Within a single pedigree, or set of small interrelated communities, there is no control population. Results based on controls chosen within the 
pedigree are confounded with the pedigree structure. We shall require our test of non-randomness to be made within the context of a given number of cases and of a given pedigree structure.

\section{Statistical methodology}

Our null hypothesis is that "the observed cases are randomly positioned within that section of a pedigree that constitutes the study population", and thus the first requirement is for a (discrete) measure of position which can be applied to any individual. The measure should divide the study population into groups of the same order of magnitude. In addition the measure will imply a distance function between individuals with different values of the measure.

Thus we have a study population of $N$ individuals, and a position measure which takes $K$ values, $m_{1}, \ldots, m_{K}$ dividing the population into $K$ cells with numbers $n_{i}$ in the $i$ th. The distance between cells $i$ and $j$ will be denoted $d_{i j}$. Thus the structure of the population is reduced to

$$
\left\{n_{1}, \ldots, n_{K}\right\} ; \quad \sum_{i=1}^{K} n_{i}=N
$$

We assume further that within the population there are $P$ "cases" and we shall label these specified individuals $a, b, c \ldots$ Their measure values we shall denote $M_{a}, \ldots$ and the distances between the cells in which they are positioned as $D_{a b}, D_{b c}, \ldots$.

We shall consider two statistics; one will be the mean value of the position measure for the cases. The second will be a measure of clustering with respect to the position measure; the sum of all observed pairwise distances $D_{a b}$. These distances are not independent, but this can be taken into account in the analysis. Nor, of course, are the positions of different individuals independent, since these constitute a sample without replacement from a finite population.

Thus we have two statistics

$$
\mu=\frac{1}{P} \sum_{a} M_{a} \quad \text { and } \quad \mathscr{D}=\sum_{a<b} \sum_{a b} .
$$

Assuming only exchangeability of the joint distribution of cases, we have

$$
\begin{aligned}
E(\mu) & =E\left(M_{a}\right), \\
E\left(\mu^{2}\right) & =\frac{1}{P}\left\{E\left(M_{a}^{2}\right)+(P-1) E\left(M_{A} M_{B}\right)\right\} .
\end{aligned}
$$

Denoting by $x^{(r)}$ the product $x(x-1) \ldots(x-r+1)$ we have also

$$
E(\mathscr{D})=\frac{1}{2} P^{(2)} E\left(D_{a b}\right)
$$

and

$$
E\left(\mathscr{D}^{2}\right)=\frac{1}{2} P^{(2)} E\left(D_{a b}^{2}\right)+P^{(3)} E\left(D_{a b} D_{b c}\right)+\frac{1}{4} P^{(4)} E\left(D_{a b} D_{c d}\right)
$$

these last coefficients being, respectively, the numbers of terms in $\mathscr{D}^{2}$ with both, one and neither indexing case-subscript common to the two distances whose product constitutes the term. 
Now under the null hypothesis of random positioning of cases

$$
\begin{aligned}
& E\left(M_{a}\right)=\frac{1}{N} \sum_{1}^{K} n_{i} m_{i} \\
& E\left(M_{a}^{2}\right)=\frac{1}{N} \sum_{1}^{K} n_{i} m_{i}^{2}
\end{aligned}
$$

and

$$
E\left(M_{a} M_{b}\right)=\left\{\sum_{i=1}^{K} n_{i}^{(2)} m_{i}^{2}+\sum_{i \neq j} n_{i} n_{j} m_{i} m_{j}\right\} / N^{(2)} .
$$

the two terms of this last expression corresponding to the two cases falling or not falling within the same cell. For the distance statistic we have

$$
E\left(D_{a b}\right)=\sum_{i \neq j} d_{i j} n_{i} n_{j} / N^{(2)}
$$

and

$$
E\left(D_{a b}^{2}\right)=\sum_{i \neq j} d_{i j}^{2} n_{i} n_{j} / N^{(2)}
$$

it being only necessary to consider the cases in different cells $(i \neq j)$ since $d_{i i}=0$. In considering $E\left(D_{a b} D_{b c}\right)$ we must encompass the two possibilities that patients $a, b, c$, are in different cells $i, j, k$, (respectively) and also the case $k=i$. Other cases of coexistence in cells provide zero products $D_{a b} D_{b c}$. Thus we have

$$
E\left(D_{a b} D_{b c}\right)=\left[\sum_{\neq(i, j, k) \neq} \sum_{i j}\left\{d_{i j} d_{j k} n_{i} n_{j} n_{k}\right\}+\sum_{i \neq j}\left\{d_{i j}^{2} n_{i} n_{j}\left(n_{i}-1\right)\right\}\right] / N^{(3)} .
$$

[The notation " $\neq(i, j, k) \neq$ " is used to denote all ordered sets $(i, j, k)$ with no pair equal.]

Finally, in considering $E\left(D_{a b} d_{c d}\right)$ we may have cases $a, b, c$, and $d$ in positions $i, j, k$, and $l$ with

$$
i=k, j=l \quad \text { or } \quad i=l, \quad j=k \quad(i \neq j)
$$

or $(k$ or $l)=(i$ or $j)$, but not both, $(i \neq j, k \neq l)$

or $(k, l, i, j)$ all different $(i \neq j, k \neq l)$.

All other combinations provide zero distance product. Thus

$$
\begin{aligned}
E\left(D_{a b} D_{c d}\right)= & \left\{\sum_{\neq(i, j, k, l) \neq} \sum_{i}\left(d_{i j} d_{k l} n_{i} n_{j} n_{k} n_{l}\right)+4 \sum_{\neq(i, j, l) \neq} \sum_{j}\left(d_{i j} d_{j l} n_{i} n_{j}^{(2)} n_{l}\right)\right. \\
& \left.+2 \sum_{i \neq j} \sum_{i}\left(d_{i j}^{2} n_{i}^{(2)} n_{j}^{(2)}\right)\right\} / N^{(4)} .
\end{aligned}
$$

Thus for any given population histogram, $\boldsymbol{n}=\left\{n_{1}, \ldots n_{k}\right\}$, we may compute the mean and variance of our statistics. The higher order moments may be computed similarly, and, in theory, the complete multitype hypergeometric distribution. However, unless $N$ is very small, this is not only not feasible but also unnecessary, a Normal approximation being sufficiently accurate for use in statistical tests. 


\section{THE NEWFOUNDLAND STUDY POPULATION}

The particular problem provoking this methodology is that of three adjacent Newfoundland communities showing many cases of a variety of lymphoreticular malignancies. This population has been described by Buehler et al. (1975), Marshall et al. (1980, and other papers). Our aim is to consider whether, given the number of cases, they are non-randomly distributed within the population. An interesting feature of this population is that 85 per cent of them are descended from a single couple, who first settled in this area of Newfoundland in 1820 , and have been denoted $J$ and $M$, (Buehler et al., 1975).

The total data file of individuals studied, and their known relatives and ancestors within the three communities, contains records of 3936 individuals. On addition of unspecified parents, for individuals one of whose parents is known, the number increases to 4028 . (This is a standard procedure to facilitate pedigree analysis.) Of these 3541 are connected to $J$ and $M$, where "connected" includes all known descent and marriage links. We restrict attention to this subset: Since all the known cases lie within it, this can only make our statistical procedures conservative. It seems desirable to split off the unconnected families, who, by this fact alone, are not typical of the communities.

Of these 3541 we take as our study population those living and resident in the communities at the time of the recent study, who were thus medically examined. In addition we include the sixteen now-dead patients diagnosed during that study and the one now-dead individual used as a control for one of these patients in previous analyses (Salmon et al., 1980). The addition of the dead patients is of course necessary for meaningful results; their deaths were the result of the disorders under investigation. The addition of the single dead control can have no impact on the results regarding the patients; he is included to allow comparisons with previous analyses. The study population thus defined consists of 1677 individuals.

Salmon et al. (1980) have recently presented a study of the distribution of patients in the population. Their main conclusions are that cases are "more closely" related to each other than are controls, that patients "appear" to derive their susceptibility from the original couple $J$ and $M$, and that they "could" do so via just four of the eight offspring of $J$ and $M$ who contribute to the current population. We shall hope to quantify some of these conclusions by constructing statistical tests.

Since we have restricted our attention to the subset of the population whose genealogy is connected to $J$ and $M$ we shall not investigate directly the degree to which the susceptibility of the cases is associated with descent from $J$ and $M$. That all cases are so descended is clearly of interest, but in fact 1512 of our 1677 individuals are so descended. Salmon et al. (1980) have noted that many more of the patients are descended via both parents than are the controls; this bilaterality of descent will be more fully analysed elsewhere.

\section{THE MARRIAGE NODE GRAPH METRIC}

To detect clustering, in the broad sense of closer social or genetic relationship between cases, a simple metric is that proposed by Edwards 
(1980). The distance between any two individuals in the pedigree is defined to be the minimum number of steps from one to the other on the marriagenode graph (Cannings et al., 1978). Choosing any particular "source" individual, $S$, we may define a coordinate for each other individual which is their distance from $S$, and a pairwise clustering with respect to this coordinate given by the absolute value of the difference in coordinate values. By choosing several such "sources" we may hope to detect a variety of patterns of clustering, since each source individual partitions the population differently, according to distances from that source. Although tests based on clustering with respect to different sources will not be statistically independent, we may obtain indications of the possible ancestral origin of any genetic predisposition. It is not necessary that the source individuals chosen be founders, but in the first instance we take three founders who maximally divide the population. That is, when coordinates with respect to all three founders are taken, the maximum occupancy of any cell of the 3-dimensional histogram is a minimum (Edwards, 1980).

The results of applying the above metric to the study population and to the sets of cases and controls are given in tables 1 and 2. The "controls" are of course no essential part of our current approach; we use them as a base point for comparison. Were they indeed random members of the population they should show no clustering as measured by our statistics. However, clustering detected by our measures could be due to age or other nongenetic demographic factors. Since controls are age-matched and sibshipsize-matched with the cases (Salmon et al., 1980) we can use them to assess the influence of these factors.

The results, both for the distance measure itself, and for clustering of that measure, are given in table 3 . There are many interesting features, although few are markedly significant. We note first that neither cases nor controls are on average atypical in their distance from the founder couple $J$ and $M, J$ being the source individual 6501 . However, this certainly does not preclude a genetic factor originating with this couple. Since so large a proportion of the population are direct descendents, the only anomaly that is likely to be detected via this source individual would be a generational or age effect. That this does not occur to any significant extent is reassuring. However, we

\section{TABLE 1}

\begin{tabular}{|c|c|c|c|}
\hline $\begin{array}{l}\text { Founder } \\
\text { distance }\end{array}$ & 6896 & $6501(J)$ & 6996 \\
\hline 2 & 4 & & \\
\hline 3 & 21 & 28 & \\
\hline 4 & 41 & 238 & 26 \\
\hline 5 & 21 & 686 & 154 \\
\hline 6 & 38 & 589 & 408 \\
\hline 7 & 162 & 102 & 403 \\
\hline 8 & 361 & 25 & 236 \\
\hline 9 & 458 & 7 & 182 \\
\hline 10 & 351 & 1 & 200 \\
\hline 11 & 181 & & 55 \\
\hline$\geqq 12$ & 39 & 1 & 13 \\
\hline Total & 1677 & 1677 & 1677 \\
\hline
\end{tabular}


CLUSTERING IN PEDIGREES

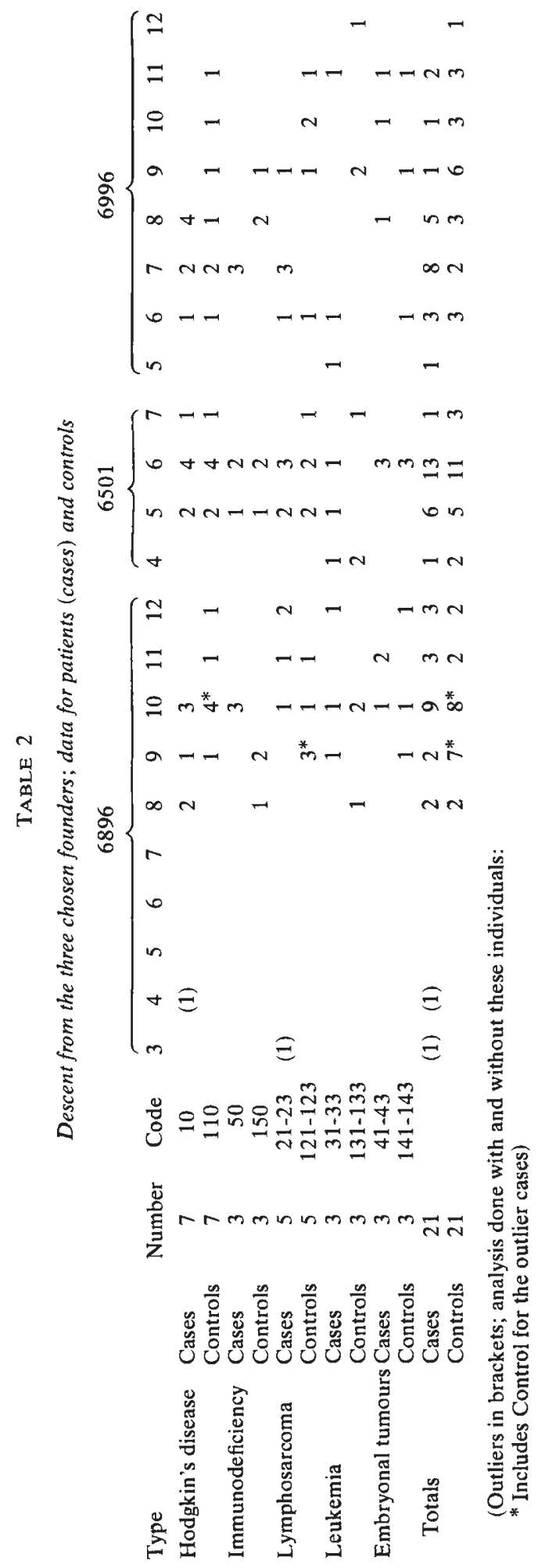


TABLE 3

Analysis of distance values

(a) Average distances

$\begin{array}{cc}\text { Founder } & \begin{array}{c}\text { Average distance } \\ \text { for cases }\end{array} \\ 6896 & 9.52 \\ 6501 & 5.67 \\ 6996 & 7.62\end{array}$

Average distance for controls

$\begin{array}{cc}\text { Expected } & \begin{array}{c}\text { Standard } \\ \text { deviation }\end{array} \\ 8.76 & 0.38 \\ 5.37 & 0.21 \\ 7.42 & 0.37\end{array}$

(b) Clustering of distances

\begin{tabular}{|c|c|c|c|c|c|}
\hline & & \multicolumn{3}{|c|}{ Observed sums of pairwise distances } & \multirow{2}{*}{$\begin{array}{l}\text { Standard } \\
\text { deviation }\end{array}$} \\
\hline & & Cases & Controls & Expected & \\
\hline \multirow{2}{*}{$\begin{array}{l}\text { Hodgkin's } \\
\text { disease }\end{array}$} & & $32(11)^{*}$ & $22(15)$ & $\begin{array}{c}38.4 \\
(27.4)\end{array}$ & $\begin{array}{c}14 \cdot 6 \\
(11 \cdot 4)\end{array}$ \\
\hline & $\left\{\begin{array}{l}6501 \\
6996\end{array}\right.$ & $\begin{array}{l}16 \\
18\end{array}$ & $\begin{array}{l}16 \\
45\end{array}$ & $\begin{array}{l}20 \cdot 8 \\
40 \cdot 2\end{array}$ & $\begin{array}{r}7 \cdot 7 \\
11 \cdot 3\end{array}$ \\
\hline $\begin{array}{l}\text { Hodgkin's } \\
\text { disease } \\
\text { +I.D. }\end{array}$ & $\left\{\begin{array}{l}6896 \\
6501 \\
6996\end{array}\right.$ & $\begin{array}{l}32 \\
30 \\
33\end{array}$ & $\begin{array}{l}44 \\
30 \\
66\end{array}$ & $\begin{array}{l}82 \cdot 3 \\
44 \cdot 6 \\
86 \cdot 2\end{array}$ & $\begin{array}{l}25 \cdot 7 \\
13 \cdot 4 \\
19 \cdot 5\end{array}$ \\
\hline \multirow[t]{2}{*}{ Totals } & 6896 & $241(212)$ & $233(226)$ & $\begin{array}{c}384 \cdot 3 \\
(347 \cdot 7)\end{array}$ & $\begin{array}{c}80 \cdot 8 \\
(74 \cdot 7)\end{array}$ \\
\hline & $\begin{array}{l}6501 \\
6996\end{array}$ & $\begin{array}{l}138 \\
346\end{array}$ & $\begin{array}{l}190 \\
424\end{array}$ & $\begin{array}{l}208 \cdot 0 \\
402.5\end{array}$ & $\begin{array}{l}41.9 \\
59 \cdot 6\end{array}$ \\
\hline
\end{tabular}

* Figures in brackets are results when the two outliers are excluded from the analysis.

see that both cases and controls are significantly more distant from the founder 6896 than a random positioning should give, and that controls are so also for the founder 6996.

The results for controls are of interest, particularly since an age-effect explanation has been precluded by their non-significance with regard to $J$ and $M$. Structural restrictions and availability of individuals may result in a carefully chosen set of matched controls being uniformly spread through a population rather than randomly. A uniform spread with respect to some structural fact (for example, descent from $J$ and $M$ ) can clearly result in a bias with respect to other factors (in particular, distance from other founders, who are chosen precisely because they partition the population differently from $J$ and $M$ ). This appears to be the explanation here, and demonstrates a danger of analyses based on matched controls within a single pedigree. Cases appear to be typical with regard to their distance from founder 6996 , but their significantly greater distance than expected, from founder 6896, may indicate an effect of descent from individuals not themselves close to this founder.

In the analysis of clustering with respect to the distance measure, there are again some interesting indications. In the case of founder 6896 , both cases and controls are more clustered than expected, but neither figure is significant. This result is a reflection of the high mean distance discussed above. In the case of founder 6996 the cases are substantially more clustered than expected and than controls, although not significantly so. This result is of interest in view of the mean distances with respect to this founder: a priori, greater clustering would have been expected for the controls, these being the more extreme. For founder $6501(J)$, cases 
definitely show greater than expected clustering whereas the controls do not, although again the result is not highly statistically significant.

In addition we have considered two subgroups of patients. The seven Hodgkin's Disease patients have attracted particular attention (Buehler et al., 1975), and indeed show greater than expected clustering, that with respect to founder 6996 being significant. The other group considered is of the seven Hodgkin's Disease patients together with three cases of Immune Deficiency. Surprisingly, it is not the previously noted clustering (Salmon et al., 1980) with respect to founder 6501 that is significant, but that for founders 6896 and 6996 . This clustering is not shown to the same extent by controls. That clustering is associated with a particular founder does not of course implicate that founder genetically. One alternative is a genetic effect in the non-founder spouse, or other relative by marriage.

Thus the above analysis has given some clear indications that there truly is some clustering effect that bears further investigation. However, it has not had the power to detect precisely whom are the individuals implicated. We now apply the same method to an alternative metric to shed some light on this problem.

\section{THE DESCENT METRIC}

Every individual of the study population is or is not descended from a specified source individual. By specifying several such sources $\left(A_{i}, 1 \leq i \leq\right.$ $k$ ) we may divide the population into $2^{k}$ subsets, $\mathscr{T}_{j}$, according to which subset $S_{j}\left(1 \leqq j \leqq 2^{k}\right)$ of $\left\{A_{i}\right\}$ the individuals are descended from. Each individual in $\mathscr{T}_{j}$ may be assigned the coordinate $m_{j}=\left|S_{j}\right|$, the number of sources ancestral to $\mathscr{T}_{j}$.

Clustering may be measured by a distance $d_{i j}$, between individuals of $S_{i}$ and $S_{j}$, given by

$$
d_{i j}=\left|S_{j}\right| S_{i}|+| S_{i}\left|S_{j}\right| \text {, }
$$

the number of sources ancestral to $\mathscr{T}_{j}$ but not $\mathscr{T}_{i}$ or vice versa. Thus using the method of section 3 we may detect both atypical degree of descent, and atypical patterns of descent.

In view of the particular structure of our population it was decided to choose as the sources the children of $J$ and $M$ (or equivalently the founders who are spouses of these children). There are eight children who contribute to the current population as shown in table 4 , and the four chosen divide the

TABLE 4

Numbers of descendants of the children of $J$ and $M$

$\begin{array}{ccc}\text { Child of } J \text { and } M & \text { Total descendants } & \begin{array}{c}\text { Total in study } \\ \text { population }\end{array} \\ 6267 & 604 & 305 \\ 7095 & 660 & 437 \\ 6455 & 117 & 26 \\ 7092 & 1780 & 1173 \\ 6606 & 727 & 518 \\ 7097 & 383 & 260 \\ 6597 & 1230 & 877 \\ 6200 & 1015 & 671\end{array}$


TABle 5

Histogram of population by descent from 7092, 6606, 6597, 6200

$\begin{array}{cc}\text { Coordinate } & 7092 \\ 0 & 0 \\ 1 & 0 \\ 2 & 0 \\ 3 & 0 \\ 4 & 0 \\ 5 & 0 \\ 6 & 0 \\ 7 & 0 \\ 8 & 1 \\ 9 & 1 \\ 10 & 1 \\ 11 & 1 \\ 12 & 1 \\ 13 & 1 \\ 14 & 1 \\ 15 & 1\end{array}$

6597
0
0
1
1
0
0
1
1
0
0
1
1
0
0
1
1

6200
0
1
0
1
0
1
0
1
0
1
0
1
0
1
0
1

Total
1149
65
331
11
91
48
43
21
341
406
310
199
46
163
213
102

Total in study population

["1" denotes descent; " 0 " no descent $]$

population as shown in table 5 . These four offspring were chosen to divide the study population most equally, independently of the attention directed upon them by Salmon et al. (1980).

The detailed data for both cases and controls, are given in table 6, and the average descent values, together with the mean and standard deviation under the null hypothesis are given in table 7. Also in table 7 are the measures of clustering with respect to descent from the four ancestors. We see that cases have a higher measure of descent from the ancestors, and controls a lower, than expected under the null hypothesis of a random positioning, although neither is highly significant. The result for controls may be attributed to a possible avoidance of close relatives of cases. Overall there is also no evidence of clustering with respect to descent from these founders; the slightly low figure for controls may again be attributed to their low mean value.

However, the values for Hodgkin's Disease and HHodgkin's disease or Immunodeficiency show significant clustering, that does not apply also to controls. In the latter case particularly there seems to be strong evidence that these members are atypical with respect to their descent from the four chosen ancestors, and more particularly with respect to the subset of the four founders from which they are descended. All are descended from 7092, and nine of the ten from 6597. This is not as expected of a random ten individuals! This result must be treated with caution. It is to some degree retrospective, being a clustering that was previously noted by Salmon et al. (1980). Nonetheless, whereas some apparent effects have not stood up to statistical analysis, this one clearly does. Whatever the conclusions regarding the wider group of lymphoreticular malignancies, this subgroup will bear much closer investigation. There are undoubtedly indications of some genetic effect, and the next stage must be to construct tests of specific genetic alternatives. 


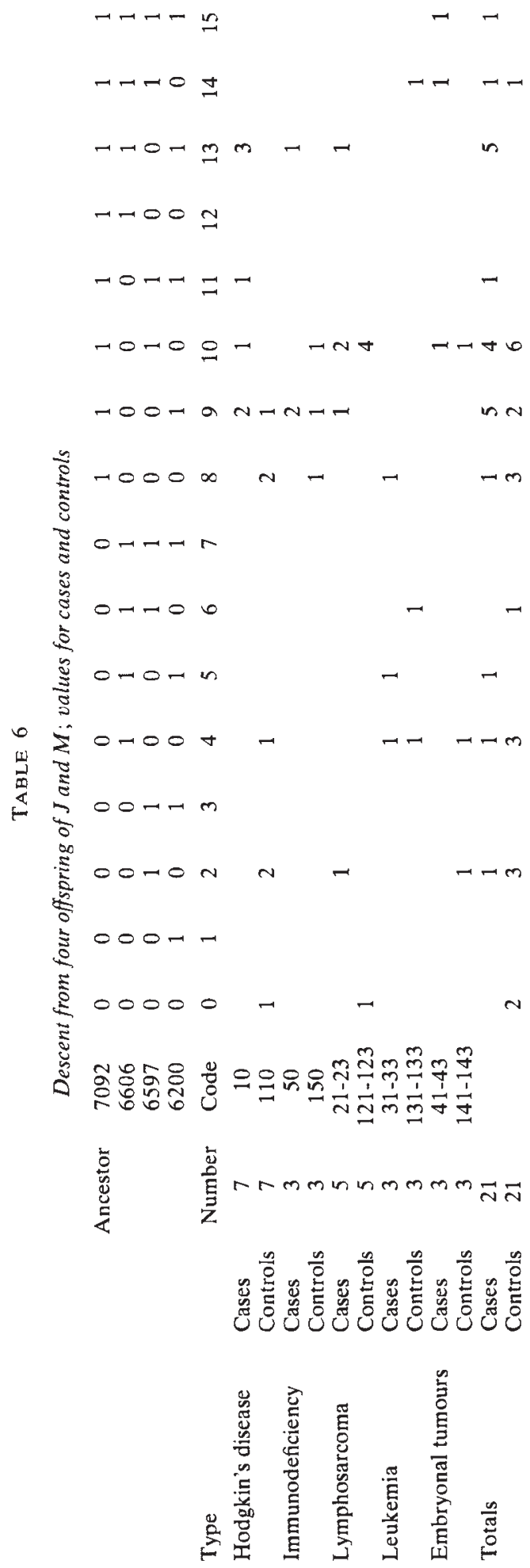


TABLE 7

Analysis of descent values

(a) Average descent [number of the four offspring descended from]

$\begin{array}{ll}\text { cases } & 2.29 \\ \text { controls } & 1.43 \\ \text { expected } & 1.93 \\ \text { standard deviation } & 0.23\end{array}$

(b) Clustering of descent

\begin{tabular}{lcccc} 
& $\begin{array}{c}\text { Observed sum of pairwise distances } \\
\text { Cases }\end{array}$ & Controls & \multicolumn{2}{c}{$\begin{array}{r}\text { Standard } \\
\text { Expected }\end{array}$} \\
Hodgkin & 28 & 34 & $38 \cdot 37$ & $5 \cdot 85$ \\
Hodgkin + I.D. & 49 & 70 & $82 \cdot 22$ & $9 \cdot 42$ \\
Total & 360 & 336 & $383 \cdot 70$ & $25 \cdot 80$
\end{tabular}

Acknowledgment.-This work arose out of a visit to Memorial University of Newfoundland in 1977. I am grateful to Dr W. J. Marshall, and others in the Faculty of Medicine, for giving me access to their data, and for their continuing interest and encouragement. The data were collected with support from the Canadian Medical Research Council and the Canadian National Cancer Institute.

\section{REFERENCES}

BUEHLER, S. K., FIRME, F., FODOR, G., FRASER, G. R., MARSHALL, W. H., AND VAZE, P. 1975. Common variable immunodeficiency, Hodgkin's disease, and other malignancies in a Newfoundland family. Lancet, 1, 195-197.

CANNINGS, C., THOMPSON, E. A., AND SKOLNICK, M. H. 1978. Probability functions on complex pedigrees. Adv. Appl. Prob., 10, 26-61.

CARMELLI, D., KARLIN, J., AND WILliAMS, R. R. 1979. A class of indices to assess major-gene versus polygenic inheritance of distributive variables. In Genetic Analysis of Common Diseases, eds. C. F. Sing and M. H. Skolnick, pp. 259-270. Alon R. Liss Inc.; New York.

EDwards, A. W. F., 1980. Parsing a genealogy. Adv. Appl. Prob., 12, 2-3.

FAIN, P. R. 1978. Characteristics of simple sibship variance tests for the detection of major loci and application to height, weight and spatial performance. Ann. Hum. Genet., 42, $109-120$.

MARSHALL, W. H., BUEHLER, S. K., CRUMLEY, J., SALMON, D., LANDRE, M. F., AND FRASER, G. R. 1980. A familial aggregate of Hodgkin's disease, common variable immunodeficiency and other malignancy cases in Newfoundland. I. Clinical Features. Clin. Invest. Med., 2, 153-159.

SALMON, D., LANDRE, M. F., FRASER, G. R., BUEHLER, S. K., CRUMLEY, J., AND MARSHALL, W. H. 1980. A familial aggregate of Hodgkin's disease, common variable immunodeficiency and other malignancy cases in Newfoundland. II. Genealogical analysis and conclusions regarding hereditary determinants. Clin. Invest. Med., 2, 175-181.

SMITH, C. A. B., LOESCH, D. Z., AND BENER, A. 1978. Search for heterozygotes in quantitative characters. Ann. Hum. Genet., 42, 121-128.

THOMPSON, E. A. 1979. Ancestral inference. III. The ancestral structure of the population of Tristan da Cunha. Ann. Hum. Genet., 43, 167-176.

THOMPSON, E. A., AND ROBERTS, D. F. 1980. Heterozygosity and kinship on Tristan da Cunha. Amer. J. Hum. Genet, 32, 445-452.

WILLIAMS, R. R., SKOLNICK, M. H., CARMELLI, D., MANESS, A. T., HUNT, S. C., HASSTEDT, S., REIBER, G. E., AND JONES, R. K. 1979. Utah Pedigree Studies Design and preliminary data for male CHD deaths. In Genetic Analysis of Common Diseases, eds. C. F. Sing and M. H. Skolnick, pp. 711-732. Alan R. Liss Inc.; New York.

WILliAMSON, J. S., BISHOP, D. T., TAVARÉ, S., AND TAVARÉ, C. J. 1979. On the independence structure that exists within a pedigree, with an application to testing for a major gene. Genetic Analysis of Common Diseases. eds. C. F. Sing and M. H. Skolnick, pp. 271-276. Alan R. Liss Inc.; New York. 